\title{
Зависимость оценки стоимости компании в сделке M\&A от типа компании-покупателя
}

\author{
Партин И.М. ${ }^{6}$
}

Оченка стоимости компании в сделках по слиянию и поглощению (сделки $M \& A$ ) может зависеть от того, кто является покупателем. Так, существует распространенное мнение о том, что стратегические инвесторы платят более высокую иену за акции компании, тогда как финансовые инвесторы стараются приобрести акиии по более низкой цене. В статье проводится регрессионный анализ с целью проверить данную гипотезу, где $в$ качестве зависимой переменной выступает мультипликатор EV/EBITDA по сделкам M\&A. По результатам анализа был сделан вывод о том, что данная гипотеза не может быть отвергнута, поэтому собственникам продаваемых компаний в первую очередь нужно обращаться к стратегическим инвесторам, которые могут заплатить более высокую цену.

$$
\text { JEL: } G 14, G 34
$$

Ключевые слова: слияния и поглощения, стоимость компании, типь инвесторов, мультипликатор, EV/EBITDA

\section{Введение}

Одним из ключевых вопросов при осуществлении сделки по продаже компании является составление списка потенциальных инвесторов. В зависимости от специфики отрасли в данный список могут попасть различные типы инвесторов, например компанииконкуренты из данной отрасли, компании из смежных отраслей (поставщики или клиенты), финансовые инвесторы (фонды прямых инвестиций, индивидуальные инвесторы), государственные компании и фонды. Все указанные типы инвесторов могут быть как из страны компании-продавца, так и из других стран, а также как публичными компаниями, так и частными.

У каждого из таких инвесторов существует свой подход к оценке стоимости бизнеса и своя требуемая доходность на вложенные средства. Существует распространенное мнение о том, что стратегические инвесторы, под которыми, как правило, понимаются компании из той же или смежной отрасли, а также государственные холдинги, обычно готовы платить более высокую цену за объект сделки. Причина заключается в том, что целью приобретения для таких инвесторов является встраивание покупаемой компании в структуру холдинга покупателя. Таким образом, компания приобретается навсегда, без цели ее дальнейшей перепродажи, а значит, срок окупаемости инвестиций в сделке может быть долгим.

Финансовые инвесторы, в свою очередь, обычно приобретают компании для того, чтобы усилиями своей управленческой команды увеличить эффективность бизнеса приобретаемой компании, нарастить финансовые показатели, повысить оценку стоимости и продать компанию стратегическому инвестору или вывести ее акции на биржу через публичное размещение. В результате требуемые сроки окупаемости инвестиций у таких инвесторов короче, а значит, они не могут позволить себе заплатить слишком высокую цену за приобретаемую компанию.

В то же время, если компания-продавец по каким-то причинам является настолько привлекательной для приобретения, что между потенциальными инвесторами возникает конкуренция, то она может изменить подход к оценке со стороны финансовых инвесторов.

\footnotetext{
${ }^{6}$ Канд. эконом. наук, преподаватель кафедры экономики и финансов фирмы НИУ ВШЭ, директор в инвестиционном банке NHCapital
} 
Так, например, если компания имеет существенный потенциал роста, то финансовые инвесторы могут заплатить более высокую цену, рассчитывая на то, что при быстром росте финансовых показателей оценка компании также будет быстро расти и сможет обеспечить требуемую доходность. Кроме того, если компания является уникальной, а барьеры для входа в отрасль велики, то финансовые инвесторы будут более уверены в том, что при перепродаже они смогут найти покупателя, даже если оценка компании будет высокой.

В данной работе проведено исследование с целью проверки гипотезы о том, что стратегические инвесторы в среднем платят более высокую цену за приобретаемые компании по сравнению с финансовыми инвесторами.

\section{Обзор научной литературы}

В данной области существует целый ряд исследований, посвященных влиянию конкуренции среди покупателей на стоимость приобретаемой компании. В таких работах помимо влияния конкуренции также анализируется способ оплаты сделки (Berkovitch, Narayanan, 1990), последовательность ценовых предложений (Hirshleifer, 1989), сопротивление менеджмента приобретаемой компании (Jennings, Mazzeo, 1993) и другие аспекты. При этом в большинстве этих работ не придается значение тому, кто является конкурирующими покупателями.

Существует также целый ряд таких работ, как, например, исследование Девентер (Dewenter, 1995), в которых анализируется различие в оценке приобретаемой компании в зависимости от того, является покупатель компанией из той же страны или иностранной компанией. В данной работе автор делает вывод о том, что не существует универсального правила и реакция рынка на национальную принадлежность покупателя зависит от характеристик каждой конкретной сделки. В других подобных работах деление покупателей также производится по страновому признаку, в то время как тип инвесторов не определяется.

Анализ типа покупателей проводится довольно редко. Так, несколько американских исследователей (Dittmar, Li, Nain, 2008) в своей работе анализируют различия в конкуренции за объект сделки с финансовыми и стратегическими инвесторами. Авторы приходят к выводу о том, что потенциальным покупателям в сделке конкурировать с финансовыми инвесторами более выгодно, так как размер премии, необходимой для победы, меньше, чем в случае со стратегическими инвесторами. Это подтверждает гипотезу о том, что стратегические инвесторы готовы платить более высокую цену за приобретаемую компанию.

Другая группа исследователей (Bargeron, Schlingemann, Stulz, Zutter, 2007) пришла к выводу, что публичные компании-покупатели в среднем платят за объект сделки премию в $55 \%$ по сравнению с частными компаниями-покупателями, которые в основном представлены фондами прямых инвестиций. При этом премия платится в основном теми публичными компаниями, акционерный капитал которых распылен и решения о сделке принимаются менеджментом, тогда как публичные компании с концентрированной структурой собственности премию в основном не платят.

Различия в ценах, которые уплачивают публичные и частные компании, проводится также в работе Бугея, Синельников (Bugeja, Sinelnikov, 2012). При этом авторы акцентируют внимание на различиях в характеристиках компаний-целей и анализируют влияние этого фактора на цену сделки.

В еще одной работе (Boone, Mulherin, 2008) отмечено, что доля сделок, в которых покупателями выступают фонды прямых инвестиций, выросла в США с 6 до 30\% от всех совершаемых сделок за период с 2003 по 2007 год. В частности, этому способствовало формирование консорциумов нескольких фондов, которые могли объединять ресурсы в борьбе за компании большого размера. В результате фонды прямых инвестиций стали чаще выигрывать в конкурентной борьбе за приобретение компаний у других типов инвесторов.

При поиске работ российских авторов наиболее часто вопросы конкуренции в сделках по слиянию и приобретению рассматриваются с точки зрения антимонопольного 
регулирования, а также через призму того, что слияния и поглощения могут сами выступать инструментом рыночной конкуренции (Чумаченко, 2007).

В то же время существуют отраслевые статьи с анализом конкуренции среди покупателей. Так, Рубинштейн (Рубинштейн, 2008) рассматривает влияние конкурентной борьбы между покупателями на цену покупки в металлургической отрасли, где рост цены составлял от 15 до 80\% от размера первоначальной оферты, что показывает смещенную оценку стоимости компании при возникновении конкурентной ситуации.

В целом же работы на тему слияний и приобретений в основном анализируют их общую эффективность для покупателей или продавцов (Хусаинов, 2008) или акцентируют внимание на различиях аспектах, как, например, характеристики приобретаемых компаний (Чиркова, Чувствина, 2011), при этом типы покупателей, как правило, не рассматриваются в работах.

\section{Обзор методов исследования}

Как известно, эффективность сделок слияния и поглощения можно оценивать следующими методами:

1. Метод анализа событий (event study) - применим только для публичных компаний.

2. Метод анализа бухгалтерской отчетности (accounting study) - применим также для непубличных компаний, по которым доступна информация о финансовых показателях.

3. Метод опроса менеджеров (clinical study) - для детального разбора небольшого количества сделок.

В настоящей статье используется метод анализа бухгалтерской отчетности, позволяющий рассматривать сделки приобретения, в том числе, частных компаний, что увеличивает потенциальный объем выборки.

По всем сделкам из выборки подсчитывается мультипликатор EV/EBITDA на момент приобретения. Помимо данного мультипликатора по каждой из компаний собирается информация о других показателях сделки, таких как размер сделки, размер приобретаемого пакета, размер доходности компании по показателю EBITDA. Кроме этого, рассчитывается также среднее значение мультипликатора EV/EBITDA по тем отраслям, в которых оперируют приобретаемые компании.

\section{Формирование и первичный анализ выборки}

Для формирования выборки были взяты данные по сделкам слияний и поглощений, проходившим во всех странах мира. В качестве источника использовалась база данных Zephyr (Bureau Van Dijk). Для получения выборки для целей настоящего анализа были использованы следующие фильтры:

1. Временной период: с 1 января 2000 года по 1 июля 2008 года.

2. Отрасль объекта сделки: все сектора кроме банковского, страхового, финансового, социального, недвижимости и коммунальных услуг.

3. Покупаемая доля: более $50 \%$.

4. Статус сделки: завершенная.

5. Тип сделки: по классификации базы данных Zephyr были взяты сделки категорий Acquisition, Institutional buy-out, Management buy-out.

6. Финансовые показатели: у приобретаемой компании известны показатели выручки и EBITDA за последний год перед осуществлением сделки.

7. Минимальный объем сделки: 10 млн долл.

8. Очистка от выбросов: мультипликатор EV/EBITDA находится в диапазоне от 1 до 20, доходность по EBITDA находится в диапазоне от 5 до $75 \%$.

В результате количество наблюдений в выборке составило 1516 сделок. Для более 
полного представления о качестве выборки ниже представлены диаграммы, описывающие основные характеристики выборки.

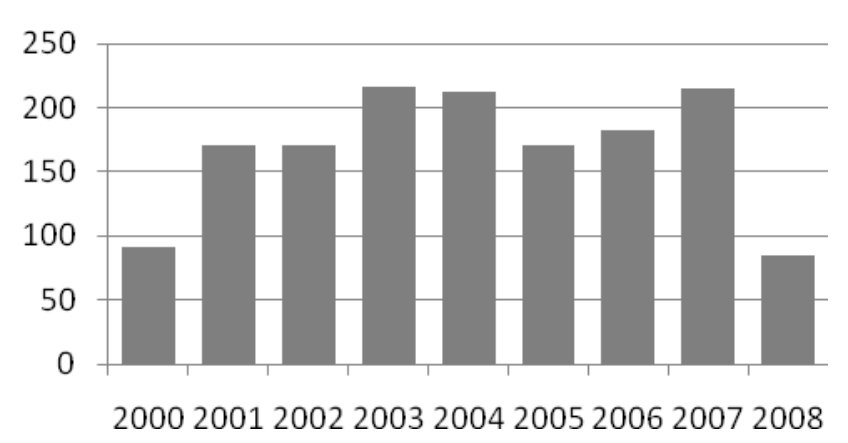

Диаграмма 1. Распределение количества сделок в выборке по годам, штук

Как видно, сделки распределились довольно равномерно по годам, за исключением начала рассматриваемого периода. Так, количество отобранных сделок в 2001-2007 годах было примерно равным, тогда как спад в 2008 году объясняется тем, что берутся сделки только в первой половине года.

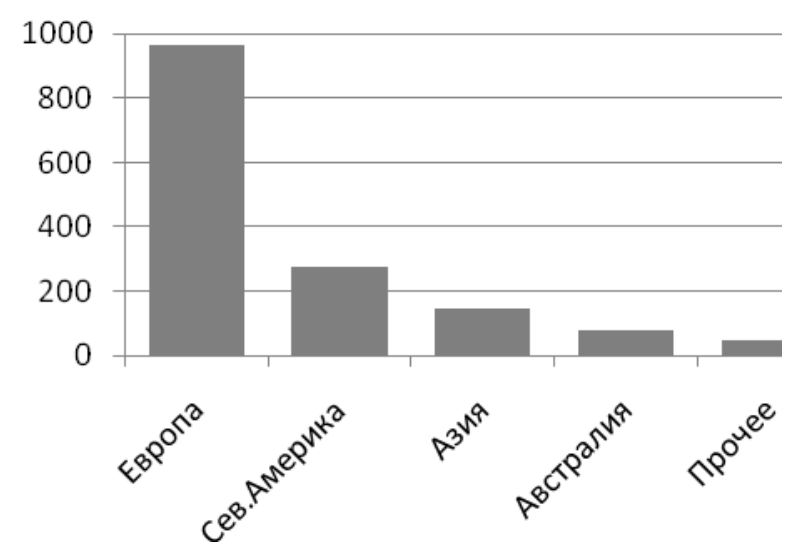

Диаграмма 2. Распределение количества сделок в выборке по регионам, штук

Распределение по регионам получилось неравномерным, со значительным весом сделок в странах Европы. Скорее всего, это связано с тем, что главный офис компании Bureau Van Dijk, поддерживающий базу данных Zephyr, находится в Брюсселе, а значит, сбор данных по странам Европы представляет более легкую задачу.

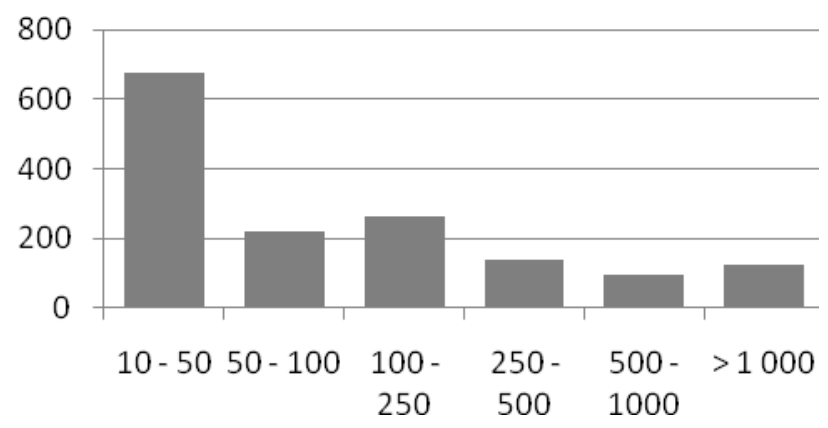

Диаграмма 3. Распределение количества сделок в выборке по размеру (млн евро), штук Распределение сделок по размеру также неравномерно, с преобладанием сделок от 10 
до 50 млн евро, которые суммарно занимают около 45\% выборки. Однако данный результат ожидаем, так как обычно количество сделок обратно пропорционально их размеру.

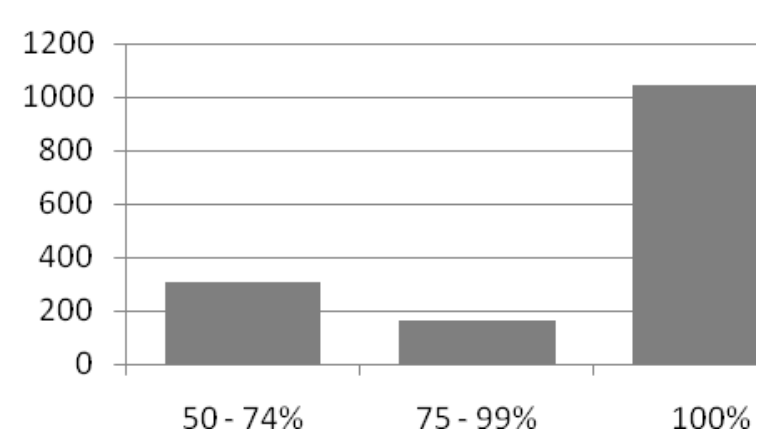

Диаграмма 4. Распределение количества сделок по размеру приобретенного пакета, штук

Как видно, большинство сделок предполагало полное приобретение компании. Однако приобретение пакета более 50\% также влечет за собой получение контроля над компанией, поэтому для целей работы рассматриваются сделки по покупке доли более $50 \%$.

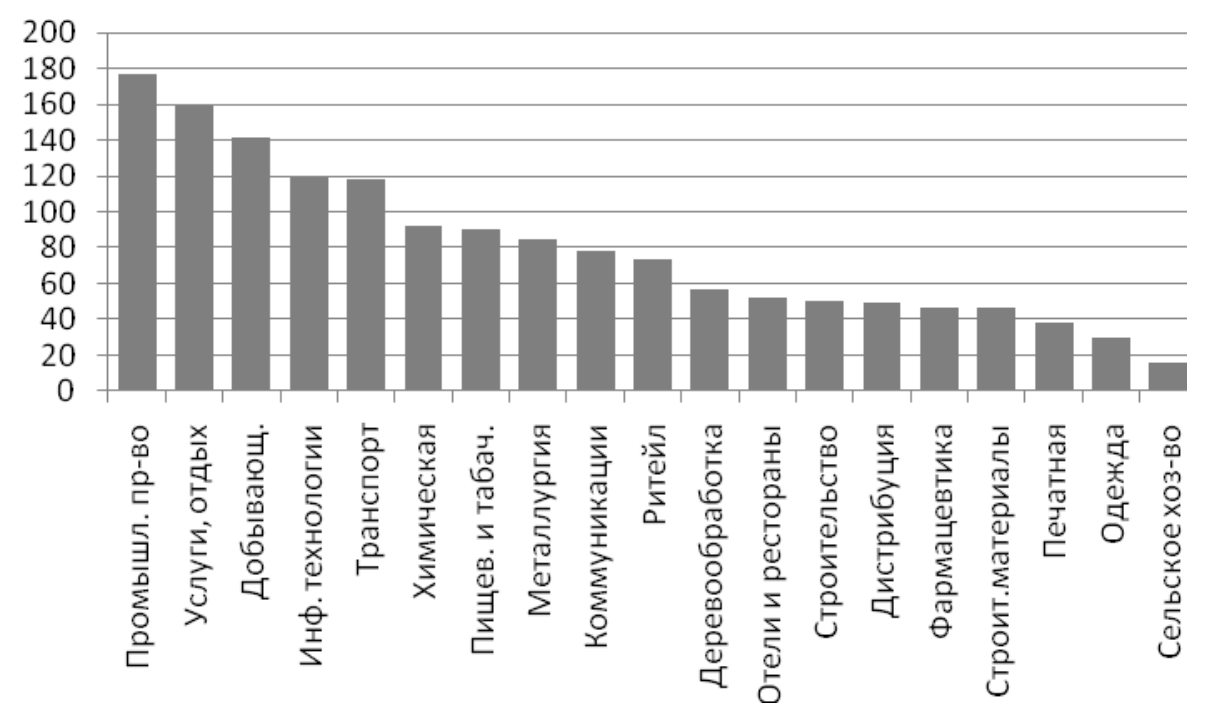

Диаграмма 5. Распределение количества сделок в выборке по отраслям, в которых работают приобретаемые компании, штук

Исходя из данных, представленных на диаграмме 5, можно сделать вывод о том, что распределение сделок между отраслями не имеет значимых выбросов в пользу тех или иных отраслей. В то же время следует отметить, что некоторые отрасли, несомненно, более полно представлены по сравнению с остальными. 


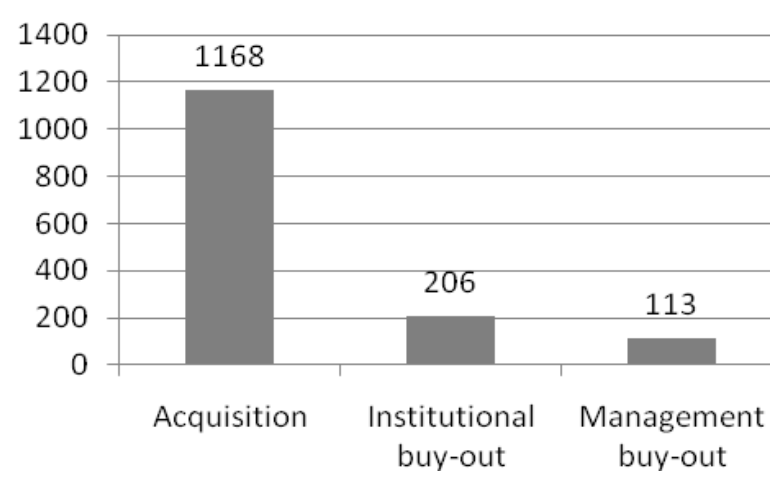

Диаграмма 6. Распределение количества сделок в выборке по типам, штук

По классификации базы данных Zephyr были отобраны следующие категории сделок: Acquisition, Institutional buy-out, Management buy-out. Под термином «Acquisition» понимается приобретение компании стратегическим инвестором; под термином «Institutional buy-out» - приобретение компании финансовым инвестором; под термином «Management buy-out» - приобретение компании со стороны группы менеджеров (либо внутренних, либо внешних по отношению к компании). Для целей настоящей работы сделки Institutional buyout и Management buy-out объединены в одну группу финансовых инвесторов, так как менеджмент часто действует как фонд прямых инвестиций - приобретает долю в компании, проводит реструктуризацию и продает компанию стратегическому инвестору.

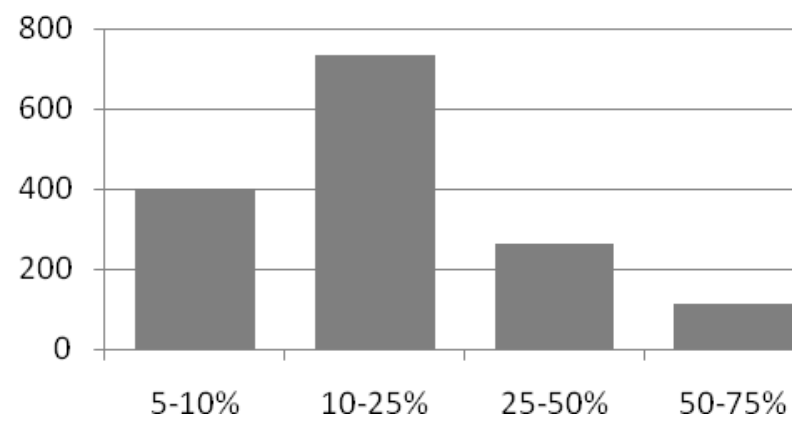

Диаграмма 7. Распределение количества сделок по доходности по EBITDA, штук

Выборка была ограничена сверху и снизу по показателю доходности по EBITDA, рассчитываемой как EBITDA/Выручка, так как вероятность ошибки внесения информации в базу данных возрастает в случае, если результирующие показатели являются слишком высокими или слишком низкими. В результате большинство сделок имеет доходность по EBITDA в размере 10-25\%, что является нормальным уровнем для большинства отраслей.

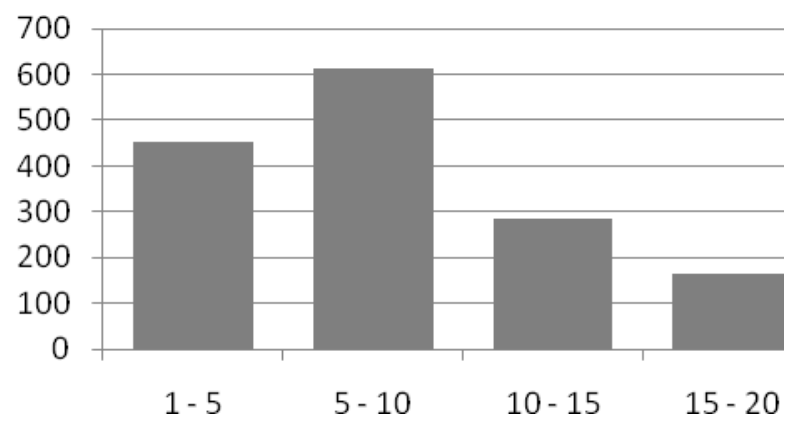

Диаграмма 8. Распределение количества сделок по мультипликатору EV/EBITDA, штук 
Выборка также была ограничена сверху и снизу по мультипликатору EV/EBITDA по той же причине, что и в случае с показателем доходности по EBITDA. В результате большинство сделок имеет мультипликатор EV/EBITDA в размере 5-10, что является нормальным уровнем для большинства отраслей. Как видно, графики распределения сделок по доходности по EBITDA и по мультипликатору EV/EBITDA похожи друг на друга. Однако это не означает, что одни и те же сделки попадают в одни и те же группы. Гипотеза о зависимости мультипликатора EV/EBITDA от доходности по EBITDA будет проверена.

\section{Построение регрессионной модели}

Основная цель данной статьи - выявить взаимосвязь между величиной мультипликатора EV/EBITDA и типом инвестора. Поэтому в качестве зависимой переменной (Multi) в модели берется мультипликатор EV/EBITDA по каждой сделке. Предполагается, что мультипликатор будет более высоким для стратегических инвесторов и более низким для финансовых инвесторов. Таким образом, основная проверяемая гипотеза в модели такова:

- Гипотеза 1. Мультипликатор EV/EBITDA по сделке зависит от типа покупателя в сделке - он выше для стратегических инвесторов и ниже - для финансовых.

В то же время на основании собранных данных можно также проверить зависимость мультипликатора EV/EBITDA от других факторов, что определяет следующие гипотезы:

- Гипотеза 2. Мультипликатор EV/EBITDA по сделке приобретения компании зависит от среднего мультипликатора в отрасли, в которой работает компания - чем выше мультипликатор в отрасли, тем выше он и для компании.

- Гипотеза 3. Мультипликатор EV/EBITDA по сделке приобретения компании зависит от доходности по EBITDA данной компании - чем выше доходность, тем выше мультипликатор.

- Гипотеза 4. Мультипликатор EV/EBITDA по сделке зависит от размера сделки, причем характер зависимости нужно определить.

- Гипотеза 5. Мультипликатор EV/EBITDA по сделке зависит от размера приобретаемого пакета акций, причем характер зависимости нужно определить.

Для проверки указанных гипотез строится регрессионная модель следующего вида:

$$
\text { Mult }_{i}=\text { InvType }_{i}+\text { AvMultInd }_{i}+\text { EBITDAmargin }_{i}+\text { LnDealSize }_{i}+\text { Stake }_{i}+\text { Const } .
$$

Независимыми переменными в модели являются следующие показатели:

- InvType - дамми-переменная, принимающая значение 1 для стратегического инвестора и 0 - для финансового;

- AvMultInd - средний мультипликатор EV/EBITDA в отрасли, в которой работает приобретаемая компания;

- EBITDAmargin - отношение EBITDA к выручке приобретаемой компании;

- LnDealSize - натуральный логарифм размера сделки;

- Stake - размер приобретаемого во время сделки пакета.

Как видно из таблицы 1 , объясняющая сила модели (коэффициент $R$-квадрат) составляет около 8\%, при этом гипотеза о значимости $F$-статистики нулю отвергается на уровне ниже 1\%. Таким образом, подтверждается зависимость мультипликатора EV/EBITDA от проверяемых переменных, однако сила данной зависимости (8\%) не очень велика и основное влияние на мультипликатор EV/EBITDA оказывают факторы, не рассмотренные в модели. В то же время гипотезы о равенстве $t$-статистик нулю по каждому из факторов, рассмотренных в модели, также отвергаются на 1\%-ном и 10\%-ном уровнях. Данный результат говорит о том, что влияние факторов, хоть и мало, но статистически значимо. 
Таблица 1

Результаты построения линейной регрессии

\begin{tabular}{|c|c|c|c|c|c|}
\hline Source & 55 & $d f$ & MS & Number of obs & \\
\hline $\begin{array}{r}\text { Madel } \\
\text { Residua } 1\end{array}$ & $\begin{array}{l}2711.53196 \\
30294.6687\end{array}$ & $\begin{array}{r}5 \\
1510\end{array}$ & $\begin{array}{l}542.306392 \\
20.0626945\end{array}$ & $\begin{array}{l}\text { Prob > F } \\
\text { R-squared }\end{array}$ & $\begin{array}{l}0.008 \\
0.08\end{array}$ \\
\hline Total & 33006.2007 & 1515 & 21.7862711 & Root MSE & 47 \\
\hline
\end{tabular}

\begin{tabular}{|c|c|c|c|c|c|c|}
\hline MLIT & coef. & std. Err. & $t$ & $P>|t|$ & [95\% conf. & Interva1] \\
\hline Invтуре & - 5296038 & .2851521 & 1.86 & 0.063 & -.0297324 & 1. 08894 \\
\hline AvMLItInd & 1.038448 & .1661377 & 6.25 & 0.000 & .712563 & 1.364333 \\
\hline EBITDA_mar $\sim-17$ & -2.814438 & .7569402 & -3.72 & 0.000 & -4.299204 & -1.329673 \\
\hline Lnoea $75 i z e$ & 60.14196 & 7.397549 & 8.13 & 0.000 & 45.6314 & 74.65252 \\
\hline stake & 2.51973 & . 6931958 & 3.63 & 0.000 & 1.160002 & 3.879459 \\
\hline _cons & -9.170702 & 1.708623 & -5.37 & 0.000 & -12.52223 & -5.819176 \\
\hline
\end{tabular}

Рассмотрим результаты проверки выдвинутых гипотез:

- Гипотеза 1. Мультипликатор EV/EBITDA по сделке действительно зависит от типа покупателя в сделке таким образом, что мультипликатор в среднем выше для стратегических инвесторов и ниже - для финансовых. Данная гипотеза является единственной из всех рассмотренных, в которой уровень значимости t-статистики составил 10\% (у остальных этот уровень ниже 1\%). Это говорит о существующей, но довольно низкой степени влияния данного параметра на мультипликатор по сравнению с другими независимыми переменными.

- Гипотеза 2. Мультипликатор EV/EBITDA по сделке приобретения компании, как ожидалось, положительно зависит от среднего мультипликатора EV/EBITDA в отрасли. Таким образом, компании в отраслях с высокими мультипликаторами EV/EBITDA могут также рассчитывать на высокий мультипликатор при сделке.

- Гипотеза 3. Мультипликатор EV/EBITDA по сделке приобретения компании вопреки ожиданиям отрицательно зависит от доходности по EBITDA данной компании - чем выше доходность, тем ниже мультипликатор. Возможно, данный результат можно объяснить тем, что если доходность компании находится на пике, то инвесторы ожидают ее снижения в будущем, что учитывается в более низком мультипликаторе.

- Гипотеза 4. Мультипликатор EV/EBITDA по сделке положительно зависит от размера сделки. Это говорит о том, что при продаже крупных компаний высокий мультипликатор более вероятен, чем при продаже небольших фирм.

- Гипотеза 5. Мультипликатор EV/EBITDA по сделке положительно зависит от размера приобретаемого пакета акций. Данную зависимость можно объяснить эффектом премии за контроль, когда за больший пакет акций покупатели готовы платить премию, так как чем больше приобретаемый пакет, тем больше прав будет иметь новый акционер.

\section{Выводы}

Несмотря на то что основной гипотезой исследования была гипотеза № 1 о зависимости мультипликатора EV/EBITDA от типа инвестора, уровень значимости данного параметра модели оказался самым низким, что говорит о существующей, но при этом небольшой силе влияния типа инвестора на мультипликатор. Частично данный результат является следствием того, что количество сделок, в которых покупателем являлись стратегические инвесторы, намного превысило количество сделок с финансовыми инвесторами. Кроме того, в разряд финансовых инвесторов попали также сделки МBO, в которых пакет акций приобретался менеджментом, что также привело к небольшой погрешности в выводах. 
На основании проверки остальных гипотез можно составить следующий список рекомендаций для собственников продаваемых компаний:

- Если компания работает в отрасли с низкими мультипликаторами, достичь высокого мультипликатора в сделке будет проблематично, так как инвесторы будут оценивать компанию, в том числе, сравнительным методом. В этом случае имеет смысл делать акцент на других видах оценки стоимости, таких как метод дисконтированных денежных потоков.

- Поскольку мультипликатор EV/EBITDA положительно зависит как от размера сделки, так и от размера пакета, наиболее выгодными будут сделки по продаже $100 \%$ акций компании в тот момент, когда она достигла пика своего развития. Таким образом, если инвесторы прибегают к частичной продаже акций в процессе развития компании, они должны быть готовы к тому, что применяемый мультипликатор в таких сделках будет ниже.

- Отдельно следует отметить полученный вывод о том, что доходность компании отрицательно влияет на мультипликатор EV/EBITDA. Возможно, данный результат можно объяснить тем, что если доходность компании находится на пике, то инвесторы ожидают ее снижения в будущем, что учитывается в более низком показателе EV/EBITDA. Поэтому рекомендация о том, что для повышения мультипликатора нужно снижать доходность по EBITDA, была бы неверной. Вместо нее можно дать рекомендацию о том, что продавать компанию можно и до достижения максимальной доходности по EBITDA, так как с учетом применения к ней более низкого мультипликатора стоимость компании может оказаться одинаковой в момент достижения максимальной доходности и за некоторое время до этого.

Подводя итог, хочется отметить, что, несмотря на указанные выше недостатки выборки, было выявлено наличие небольшого, но значимого влияния типа инвестора на мультипликатор EV/EBITDA. Данное влияние говорит о том, что собственникам продаваемых компаний следует в первую очередь искать покупателей среди стратегических инвесторов, которые в среднем будут готовы приобрести компанию исходя из оценки по более высокому мультипликатору EV/EBITDA.

\section{Список литературы}

1. Рубинштейн Т.Б. Опыт и уроки осуществления слияний и поглощений в металлургии // Металлург,. 2008. №11. С. 10-13.

2. Хусаинов 3.И. Оценка эффективности сделок слияний и поглощений: интегрированная методика // Корпоративные финансы 2008. № 1. C. 12-33. URL: http://ecsocman.hse.ru/hsedata/2010/12/31/1208181304/5_husainov_12_33.pdf.

3. Чиркова Е.В., Чувствина Е.В. Реакция рынка на объявление о приобретении компаний открытого и закрытого типов // Корпоративные финансы. 2011. № 3. C. 30-43. URL: http://ecsocman.hse.ru/hsedata/2011/11/07/1272278422/CFJ19_30_43_Чиркова_Чувствин a_.pdf

4. Чумаченко А.А. Слияния и поглощения как фактор конкуренции в российском банковском секторе // Современная конкуренция. 2007. № 5.С. 110-118.

5. Bargeron, L., Schlingemann, F., Stulz, R.M., Zutter, C. (2007), Why do private acquirers pay so little compared to public acquirers?, Working Paper № 13061: http://www.nber.org/papers/w13061.

6. Berkovitch, E., Narayanan, M.P., (1990), Competition and the medium of exchange in takeovers. Review of Financial Studies 3 (1990) 153-174.

7. Boone, A.L., Mulherin J.H. (2008), Do Private Equity Consortiums Impede Takeover Competition?: http://ssrn.com/abstract=1104224.

8. Bugeja, M., Sinelnikov, K. (2012), Public versus private takeovers of stock exchange listed targets, Australian Journal of Management, published online before print (2012).

9. Dewenter, K.L. (1995), Does the Market React Differently to Domestic and Foreign Takeover Announcements? Evidence from the U.S. Chemical and Retail Industries, Journal of Financial Economics 37 (1995) 421-441. 
10. Dittmar, A.K., Li, D., Nain, A. (2008), The Bright Side of Bidder Competition: http://ssrn.com/abstract $=1317829$.

11. Hirshleifer, D., Png, I.P.L. (1989), Facilitation of competing bids and the price of a takover target, Review of Financial Studies 2 (1989) 587-606.

12. Jennings, R.H., Mazzeo, M.A. (1993), Competing bids, target management resistance, and the structure of takeover bids, Review of Financial Studies 6 (1993) 883-909. 